\title{
Retrospective Analysis of Risk Factors for Related Complications of Chemical Ablation on Hypertrophic Obstructive Cardiomyopathy
}

\author{
Cheng-Yang $L i^{i \oplus}$ e Yun-Qi Shie \\ Department of Cardiology - Liaoning Provincial Jin Qiu Hospital, ${ }^{1}$ Liaoning Province - China \\ Department of Cardiology - Liaoning Provincial People's Hospital, ${ }^{2}$ Liaoning Province - China
}

\begin{abstract}
Background: The analysis of risk factors for predicting related complications has not been reported to date.
\end{abstract}

Objective: This study aims to investigate the risk factors of related complications of percutaneous transluminal septal myocardial ablation (PTSMA) for hypertrophic obstructive cardiomyopathy (HOCM) retrospectively.

Method: Clinical data, and one-year follow-up results of patients with HOCM, who underwent PTSMA between January 2000 and July 2013 in the Department of Cardiology, Liaoning Province People's Hospital, Liaoning Province, China, were retrospectively analyzed to determine risk factors for operative complications with multiple logistic regression analysis. All p values are two-sided, with values of $p<0.05$ being considered statistically significant.

Results: Among 319 patients with HOCM, PTSMA was performed in 224 patients (120 males and 104 females, mean age was $48.20 \pm 14.34$ years old). The incidence of PTSMA procedure-related complications was $36.23 \%(66 / 224)$, which included three cardiac deaths, two cardiac shocks, one ST-segment elevated myocardial infarction, two ventricular fibrillations, 20 third-degree atrioventricular (AV) blocks (four patients were implanted with a permanent pacemaker (PPM)), 32 complete right bundle branch blocks, two complete left bundle branch blocks, and four puncture-related complications. After multivariate logistic regression analysis, it was found that age, gender, coronary artery diseases, diabetes, heart rate, cardiac function on admission, the number of septal ablations, and the volume of alcohol were not independent risk factors correlated to the whole complications, except for hypertension (OR: 4.856; 95\% CI: 1.732-13.609). Early experience appears to be associated with the occurrence of complications.

Conclusion: Hypertension was an independent risk factor for PTSMA procedure-related complications. It might be much safer and more efficient if PTSMA procedures are restricted to experienced centers, according to the analysis results for the learning curve. (Arq Bras Cardiol. 2019; 112(4):432-438)

Keywords: Cardiomyopathy, Hypertrophic/prevention and control; Myocardial, Percutaneous Transluminal Septal Myocardial Ablation (PTSMA); Ventricular Dysfunction Left/complications.

\section{Introduction}

Hypertrophic obstructive cardiomyopathy (HOCM) is defined as primary myocardial hypertrophy with dynamic left ventricular outflow tract (LVOT) obstruction and diastolic dysfunction of left ventricle (LV). HOCM, which induces symptoms of angina, dyspnea and syncope, is a genetically determined disorder caused by mutations in genes encoding sarcomeric contractile proteins. ${ }^{1}$ Myectomy has been proven to be capable of improving short- and middle-term survival in patients with $\mathrm{HOCM}$ with severe drug refractory symptoms. ${ }^{2,3}$ With the development of techniques and equipment for percutaneous coronary intervention, percutaneous transluminal

Mailing Address: Cheng-Yang Li

NN. 317 XIAONAN Street. 110016, Shenhe District, Shenyang City, Liaoning Province - China

E-mail: chengyanglisvip@163.com

Manuscript received March 23, 2018, revised manuscript July 19, 2018, accepted August 15, 2018

DOI: $10.5935 / a b c .20190060$ septal myocardial ablation (PTSMA) has become an alternative to myectomy with a decade history., ${ }^{4,5}$ However, despite the advances in the judgment of indication, operating skill, optimal medical treatment and the management of complications, PTSMA-related complications remain high during the perioperative period. The most common complication is right bundle branch block. The most significant complications include high-degree conduction block needing PPM, acute myocardial infarction, cardiac shock, cardiac death, puncture site complications. ${ }^{6-8}$ Unfortunately, only predictors for long follow-up outcome have ever been analyzed., 7 The analysis of risk factors for predicting related complications have not been reported to date. In this report, we attempted to identify risk factors related to PTSMA procedure complications by conducting a retrospective review of 319 HOCM patients.

\section{Methods}

The study population comprised 319 patients with HOCM, who were referred to the Cardiology Department of Liaoning Provincial People's Hospital of China, and considered septal reduction therapy with PTSMA between 
January 2000 and July 2013. Among these 319 patients, 258 met the inclusion criteria for septal reduction therapy with resting left ventricular outflow tract gradient (LVOTG) $\geq 30 \mathrm{mmHg}$ or exercise-induced LVOTG $\geq 50 \mathrm{mmHg} .{ }^{9}$ The diagnostic criteria for HOCM were end-diastolic wall thickness $>15 \mathrm{~mm}$, and a non-dilated LV with an ejection fraction (EF) of $\geq 50 \% .{ }^{10}$ Exclusion criteria were hypertrophy from other causes $(n=17)$, history of myocardial infarction $(n=10)$, and prior intervention with PTSMA $(n=7)$ or septal myectomy $(n=0)$. Patients with coronary artery disease (CAD) (coronary artery stenosis $\geq 50 \%$ evaluated at coronary angiography) without myocardial infarction, mild or moderate valvular heart disease unrelated to HOCM, and patients on antihypertensive therapy were not excluded.

Diabetes was defined according to the guidelines. ${ }^{11}$ Hypertension was defined as either a systolic or diastolic elevation of blood pressure (>140/90 mmHg) or ongoing antihypertensive therapy. Hypercholesterolemia was defined as a total cholesterol level $>5.0 \mathrm{mmol} / \mathrm{L}$, or current treatment with lipid-lowering medications. The present study complied with the Declaration of Helsinki. The Local Ethics Committee of the Liaoning Province Hospital approved the study protocol, and all patients provided an informed consent.

\section{Echocardiography}

Before and during the procedure, and at follow-up, all patients underwent transthoracic echocardiography using a Hewlett-Packard Sonos 1500 (Hewlett-Packard Co., Andover, Massachusetts, USA) echocardiograph with an interfaced 2.5-MHz transducer. The following parameters were measured: intra-ventricular septum (IVS) thickness, left ventricular posterior wall (LVPW) thickness, and end-systolic and end-diastolic dimensions from the minor axis M-mode of the LV obtained from a 2-dimensional standard left parasternal view. LVOTG was measured by a continuous-wave Doppler probe positioned at the cardiac apex. EF was automatically calculated.

\section{Dobutamine stress echocardiography}

Dobutamine was administered using an infusion pump at a starting rate of $5 \mu \mathrm{g} \cdot \mathrm{kg}^{-1} \cdot \mathrm{min}^{-1}$, with increments of $5 \mu \mathrm{g} \cdot \mathrm{kg}^{-1} \cdot \mathrm{min}^{-1}$ every three minutes to a maximum dose of $40 \mu \mathrm{g} \cdot \mathrm{kg}^{-1} \cdot \mathrm{min}^{-1}$ if necessary. The stress-induced echocardiography endpoint is the end of the eighth stage of the dobutamine protocol, or following chest pain, dyspnea, a drop in arterial pressure of $20 \mathrm{mmHg}$ or more, and an ST-segment shift of $1 \mathrm{~mm}$ or more.

\section{Cardiac catheterization, gradient determination and ablation procedure}

The right femoral and right radial arteries, as well as the right femoral vein, were cannulated using the standard Judkins technique. After an intravenous bolus of 100-150 U/kg of heparin, a $6 \mathrm{~F}$ temporary pacemaker lead was placed in the right ventricle, a $6 \mathrm{~F}$ pigtail catheter was positioned in the left ventricular apex, and a $6 \mathrm{~F}$ Judkins guiding catheter was placed in the left coronary artery. The resting LVOTG was determined by simultaneous pressure recording. Provocation after premature ventricular contraction caused by the pigtail catheter (Terumo,
Japan) was performed if necessary. The adequate septal branch was identified on the coronary angiogram; after that, a 0.014-inch guide wire (Bmw; Bebi Inc., India) was inserted into the septal branch. Then, an over-the-wire balloon catheter $(1.5 / 2.0 / 2.5 \mathrm{~mm}$ in diameter, $10 / 20 \mathrm{~mm}$ in length; Medtronic, USA) was placed in the proximal part of the septal branch. After balloon inflation at 2-6 atm, the correct balloon position was determined by injection of the contrast medium via the guiding catheter into the left coronary artery, and by injection through the balloon catheter shaft into the septal branch. In order to determine the target septal branch, probationary balloon inflation and/or myocardial contrast echocardiography (MCE) was performed. When sufficient decrease in LVOTG was observed through probationary balloon inflation, the septal branch was identified as the target. MCE was routinely performed according to routine methods. ${ }^{12}$ When the target septal branch was determined, after intravenous administration of $5 \mathrm{mg}$ of diamorphine, 1-2 $\mathrm{ml}$ of absolute alcohol was slowly injected through the balloon catheter shaft. Ten minutes later, the balloon was deflated and the contrast medium was injected via the guiding catheter to ascertain that the septal branch was completely blocked. Patients were monitored in the Coronary Care Unit for three days after removal of the vascular sheaths. After 48 hours, when the patient appeared to have normal cardiac conduction, the temporary pacemaker was removed. If high-grade AV conduction disturbances were observed during the following days, PPM implantation was offered. Patients were discharged and followed at our outpatient clinics.

\section{Complications}

Complications during PTSMA procedures and in-hospital monitoring were registered. The events were acute heart failure, cardiac shock, cardiac death and arrhythmic events (bradycardia, asystole, sustained and non-sustained ventricular tachycardia, and ventricular fibrillation). Coronary artery complications were coronary dissection, coronary perforation, acute myocardial infarction, acute pericardial effusion, pericardial tamponade, and alcohol displacement. Bundle branch block and AV block including advanced heart block, which lead to PPM implantation, were registered. Advanced heart block was defined as bifascicular block, and second- or third-degree AV block. Asystole due to third-degree AV block was classified as third-degree AV block (i.e. advanced heart block). Puncture related complications were also included for the final analysis.

\section{Patient follow-up}

Patients were carefully monitored in the Coronary Care Unit for at least three days after the procedure, and back-up pacing was continued via the femoral vein when necessary. In-hospital assessment was performed for all clinical outcomes, including hemorrhagic and vascular complications (femoral artery pseudoaneurysm and puncture hematoma were also included for analysis). After discharge, monthly clinical follow-up examinations were conducted on an outpatient basis, in order to monitor the occurrence of adverse events. Examinations through catheterization were not routinely performed, and were only conducted when residual or recurrent symptoms were observed after discharge. PTSMA was repeated when necessary. A failed outcome after PTSMA was defined as the need for 
re-intervention due to the absence of clinical improvement, or recurrence of symptoms, and significant LVOTG. To analyze the influence of the learning curve in relation to PTSMA complications, patients were separated into three chronological groups (early experience: from 2000 to 2004; intermediate experience: from 2005 to 2009; late experience: from 2010 to 2013) according to their experience with PTSMA.

\section{Statistical analysis}

All data analysis was performed with the SPSS System (version 19.0; SPSS Inc., Chicago, IL, USA).One-Sample Kolmogorov-Smirnov test, and Levene's test had been used to test the normality distribution and variances equality of data. Data with normal distribution were expressed as mean \pm standard deviation (SD). Differences between groups were analyzed for statistical significance using the unpaired Student's t-test. Frequency was compared using Chi-squared $\left(\mathrm{X}^{2}\right)$ test. Multivariate stepwise logistic regression was used to select independent variables. A p-value $<0.05$ (2-tailed) was considered statistically significant.

\section{Results}

A total of 224 subjects, who were between 9-82 years old, were included into this study for final analysis (Figure 1). The detailed patients' demographic and echocardiographic characteristics were shown in Table 1.

Acute results

\section{Changes to hemodynamic results during the intervention}

An 82 year-old patient died during the injection of alcohol for acute pericardial tamponade. A mean of $1.17 \pm 0.45$ (range: 1-2) septal branches were occluded by injection of $2.07 \pm 0.89 \mathrm{ml}$ (range: $0.5-3.0 \mathrm{ml}$ ) of alcohol. A reduction in LVOTG was achieved for all patients. The mean systolic pressure difference in LVOTG at rest decreased from $67.91 \pm 37.23$ to $16.24 \pm 19.13(\mathrm{p}<0.01)$. The post premature gradient was reduced from $119.42 \pm 38.44$ to $40.83 \pm 22.61(p<0.01)$.

\section{Improvement of clinical course}

All patients complained of marked chest pain during alcohol injection, and a feeling of discomfort in the left thorax lasted up to 30 hours (10-30 hours). Clinical symptoms greatly improved in 190 patients (85\%). Differences in New York Heart Association functional class (from $1.08 \pm 0.36$ to $1.01 \pm 0.09$ ) were not statistically significant.

\section{Complications}

Two patients developed ventricular fibrillation after alcohol injection, and sinus rhythm was restored by $200 \mathrm{~J}$ of defibrillation. Two patients had cardiac shock due to the prolonged occlusion of the septal arteries. One case of thrombosis in the left anterior descending artery during the PTSMA procedure was observed. The patient was successfully treated, and coronary flows were normalized. Temporary right bundle branch block and left bundle branch block occurred in 32 patients and two patients, respectively. Furthermore, 20 patients developed a third-degree AV block, but only four patients developed a complete AV block, requiring PPM implantation. Puncture related complications occurred in four patients (femoral artery pseudoaneurysm in two patients and puncture hematoma in two patients), which were successfully treated with compression bandage.

\section{One-year noninvasive follow-up}

None of the patients were lost to follow-up. No other complications or severe major adverse cardiac events

Table 1 - Clinical characteristic of 224 patients with hypertrophic obstructive cardiomyopathy (HOCM) on admission

\begin{tabular}{lc}
\hline & Patients on admission ( $\mathbf{n = 2 2 4})$ \\
\hline Age (years) & $48.20 \pm 14.34$ \\
Male/female & $120 / 104$ \\
New York Heart Association functional class & $1.08 \pm 0.36$ \\
CAD & 13 \\
Hypertension & 47 \\
DM & 3 \\
Stroke & $1 /$ \\
HR (beats/min) & $70.92 \pm 11.66$ \\
LVOTD (mm) & $9.38 \pm 2.52$ \\
EF & $0.65 \pm 0.07$ \\
\hline CAD: coronary artery disease; DM: diabetes mellitus; HR: heart rate; \\
LVOTD: left ventricular outflow tract diameter; EF: ejection fraction.
\end{tabular}

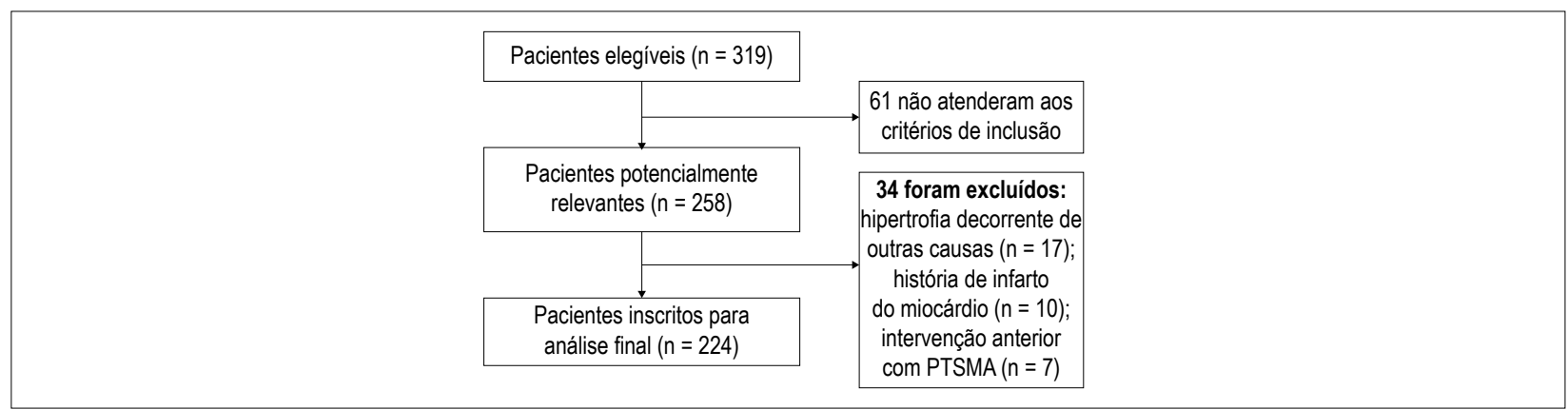

Figure 1 - Flow diagram of the patients selection. 
occurred during the clinical follow-up, except that PTSMA was successfully performed again in one patient for recurrent angina pectoris.

The univariate analysis of risk factors for PTSMA-related complications is shown in Table 2. Age, female gender, alcohol volume, the number of septal ablations, comorbidities with CAD, hypertension, and diabetes mellitus (DM) were associated with increased occurrence of complications. Results of the multiple logistic regression analysis are presented in Table 3. In multiple logistic models, except for hypertension (OR: 4.856; 95\% Cl: 1.732-13.609), age, gender, alcohol volume, the number of septal ablation, comorbidities with CAD, and DM were not potential risk factors for predicting PTSMA-related complications.

Table 4 shows the comparisons of clinical characteristics between patients with and without history of hypertension. Female patients appeared to have more cardiovascular risk factors such as hypertension, aging, DM, and history of heart failure in the present study.

As listed in Table 5, patients were chronologically divided into three groups according to their experience with PTSMA. In addition, in-hospital complications were more frequent in patients who underwent PTSMA procedures in the early stage (from 2000 to 2004), and less often in patients who unerwent PTSMA procedures later and in experienced time periods (2010-2013) $(p=0.022)$.

\section{Discussion}

PTSMA is a nonsurgical technique to reduce septal mass by producing a septal infarction using the catheter techniques reported by Sigwart. ${ }^{4}$ Permanent septal necrosis is created through the injection of alcohol in the septal branches that supply the myocardium and are responsible for LVOT obstruction induced by abnormal structure and function. This effectively reduces the pressure gradients in patients with HOCM. This technique has the advantage of micro-trauma and high success rate, as well as low mortality $(0-1.8 \%) .{ }^{13,14}$ In the present cohort, a successful reduction in LVOTG was achieved in the majority of patients during the procedure (85\%) (at rest: from $67.91 \pm 37.23$ to $16.24 \pm 19.13 \mathrm{mmHg}$, $\mathrm{p}<0.01$; post premature beat: $119.42 \pm 38.44$ to $40.83 \pm 22.61 \mathrm{mmHg}$, $\mathrm{p}<0.01)$ and at discharge (96\%).

However, the occurrence of PTSMA procedure-related complications was notable. In the present study, $14.35 \%$ $(32 / 223)$ of patients had transient complete bundle branch block (32/223), and $0.90 \%$ (2/223) of patients had complete left bundle branch block. This phenomenon was consistent with a previous report. ${ }^{15}$ The right bundle is usually supplied by proximal septal perforators. Thus, PTMSA frequently leads to complete right bundle branch block. Furthermore, septal myectomy causes complete left bundle branch block in most patients. This is the reason why PTMSA caused more complete right bundle branch and less complete left bundle branch block in our study. Not more than $10 \%$ of patients had a high-degree AV block. However, PPM was only performed in four patients (1.79\%), which was superior when compared with other PTSMA centers (46\% and 38\%). ${ }^{16,17}$ Serious complications were not uncommon. Except for patient's death from acute tamponade during the procedure, the most significant complication of the procedure was heart block, ${ }^{9}$ which led to the deaths of two patients in our study. One patient had acute severe left ventricular dysfunction during the procedure, while the other patient had heart failure during monitoring in Coronary Care Unit. In addition, reports of in-hospital ventricular fibrillation in relation to PTSMA have attracted considerable attention. ${ }^{18,19}$ We found two (0.89\%) cases of in-hospital ventricular fibrillation. According to our experience, careful monitoring was indispensable to reducing cardiac adverse events caused by ventricular arrhythmia. As for other serious nonfatal complications, acute myocardial infarction, which was caused by the spill of alcohol into the left anterior descending coronary artery, occurred in one patient. However, no coronary dissection and nonfatal cardiac tamponade occurred.

Table 2 - Univariate analysis of risk factors for related complications of PTSMA

\begin{tabular}{|c|c|c|c|}
\hline & Complications $(n=66)$ & No Complications ( $n=158$ ) & $p$ value \\
\hline Age (years) & $51.27 \pm 14.13$ & $46.91 \pm 14.28$ & 0.038 \\
\hline Male/female & $27 / 39$ & $92 / 66$ & 0.000 \\
\hline $\begin{array}{l}\text { New York Heart } \\
\text { Association functional class }\end{array}$ & $1.10 \pm 0.40$ & $1.08 \pm 0.33$ & 0.566 \\
\hline$C A D$ & 5 & 8 & 0.000 \\
\hline Hypertension & 19 & 27 & 0.000 \\
\hline DM & 3 & 0 & 0.000 \\
\hline Stroke & 1 & 0 & 0.122 \\
\hline HR (beats/min) & $71.55 \pm 11.92$ & $71.08 \pm 12.29$ & 0.792 \\
\hline LVOTD (mm) & $9.13 \pm 2.64$ & $9.33 \pm 2.54$ & 0.604 \\
\hline EF & $0.63 \pm 0.13$ & $0.66 \pm 0.08$ & 0.506 \\
\hline Alcohol volume & $2.14 \pm 0.88$ & $1.85 \pm 0.91$ & 0.023 \\
\hline Number of ablation septal & $1.19 \pm 0.43$ & $1.07 \pm 0.27$ & 0.034 \\
\hline
\end{tabular}

CAD: coronary artery disease; DM: diabetes mellitus; HR: heart rate; LVOTD: left ventricular outflow tract diameter; EF: ejection fraction. 
Table 3 - Multivariate logistic regression for potential risk factors for PTSMA complications

\begin{tabular}{lccc}
\hline & p value & Odds ratio & Cl \\
\hline Age (years) & 0.767 & 0.995 & $0.959-1.031$ \\
Male & 0.198 & 0.527 & $0.198-1.399$ \\
CAD & 0.761 & 0.761 & $0.132-4.407$ \\
Hypertension & 0.003 & 4.856 & $1.732-13.609$ \\
DM & 0.176 & 6.620 & $0.428-12.527$ \\
Alcohol volume & 0.385 & 0.757 & $0.403-1.420$ \\
Number of ablation septal & 0.436 & 0.682 & $0.370-2.253$ \\
\hline
\end{tabular}

$C A D$ : coronary artery disease; $D M$ : diabetes mellitus.

Table 4 - Comparisons of clinical and echocardiographic characteristics between patients with and without hypertension

\begin{tabular}{lccc}
\hline & Hypertension $(\mathrm{n}=\mathbf{4 6})$ & No hypertension $(\mathrm{n}=\mathbf{1 7 8})$ & $\mathrm{p}$ value \\
\hline Age & $58.13 \pm 10.10^{* *}$ & $45.23 \pm 13.95$ & 0.000 \\
Female & $28^{* *}$ & 75 & 0.025 \\
DM & $3^{* *}$ & 0 & 0.007 \\
CAD & 5 & 8 & 0.000 \\
History of heart failure & $7^{*}$ & 8 & 0.010 \\
LVOTD (mm) & $9.56 \pm 2.76$ & $9.24 \pm 2.62$ & 0.480 \\
HR (beats/min) & $70.57 \pm 11.13$ & $71.37 \pm 12.46$ & 0.692 \\
EF & $0.63 \pm 0.07$ & $0.65 \pm 0.07$ & 0.113 \\
\hline
\end{tabular}

CAD: coronary artery disease; DM: diabetes mellitus; HR: heart rate; LVOTD: left ventricular outflow tract diameter; EF: ejection fraction.

Table 5 - In hospital complications and late interventional failure according to experience

\begin{tabular}{lccc}
\hline & Early experience $(\mathbf{n}=\mathbf{7 5})$ & Intermediate experience $(\mathbf{n = 9 3})$ & Late experience $(\mathbf{n = 5 6})$ \\
\hline Events & 31 & 22 & 13 \\
No events & 44 & 71 & 43 \\
\hline
\end{tabular}

Patients' demographic characteristics should be the potential risks for PTSMA procedure complications. However, according to a previous report, ${ }^{18}$ none of the studied baseline echocardiographic, clinical and PTSMA-related characteristics were useful in predicting the PTSMA success rate and its complications. A report on nine-year follow-up results from the SZEGED study revealed that coronary flow velocity reserve (CFR) was an independent predictor of cardiovascular event-free survival for patients with HOCM..$^{13}$ However, CFR was estimated by transesophageal echocardiography, which is inconvenient in clinical practice. In the univariate analysis of our study, age, gender, alcohol volume, the number of septal ablations, comorbidities with CAD, hypertension and DM appeared to be associated with the increased occurrence of complications. However, only hypertension, and not the other characteristics, was shown to be a potential factor for predicting complications (OR: 4.856; 95\% Cl: 1.732-13.609) after multivariate logistic regression analysis. Patients with hypertension were older, showed more significant changes in the echocardiogram, presented with more comorbidities (Table 4). We might present a hypothesis that patients with hypertension had lower cardiac reserve function due to more cardiovascular risks in this cohort. Hence, this might be the reason why hypertension could be a potential risk factor for PTSMA complications.

It is well-known that clinical experience influences the results of a procedure. Similar to results observed from percutaneous coronary interventions, ${ }^{20}$ a high-volume load for operators and institutions has been proven to be associated with better procedural outcomes. The importance of a learning curve for PTSMA was confirmed in our study, because a high incidence of late PTSMA failure was noted in the early experience group of patients, whereas this number was significantly reduced with higher experience. At a frequency of approximately 16 treated patients per year, the incidence of late PTSMA complication has been reduced from $41.33 \%$ 
to $23.21 \%$, which was not different from that in other experienced centers. ${ }^{7,21}$ Therefore, PTSMA procedures might be safer and more efficient in experienced centers.

\section{Limitations}

There were several important limitations in the present study. (1) One of the most important limitations was that only a limited number of HOCM patients were examined. (2) In this study, these PTSMA procedures were elected based on the preferences of patients and the physician. Therefore, patients were not consecutively enrolled. If the patient was an older person, or had significant comorbidity conditions, PTSMA was not that strongly suggested. For older patients, especially those with concomitant disease and without enough insurance, medication or a less aggressive approach of PTSMA might be a better or the only choice, even with the incomplete elimination of LVOT obstruction. (3) The decision regarding the target septal artery was taken based on available angiographic images, as well as the assistance of MCE. However, we still could not rule out that more targeted imaging might have yielded more anatomically correct values.

\section{Conclusion}

In summary, PTSMA was effective in reducing LVOTG in HOCM patients. Hypertension was the only independent risk factor for PTSMA procedure-related complications after multivariate logistic regression analysis. In addition, PTSMA procedures might be safer and more efficient in

\section{References}

1. Kimura A. Contribution of genetic factors to the pathogenesis of dilated cardiomyopathy: the cause of dilated cardiomyopathy: genetic or acquired? (genetic-side). Circ J. 2011;75(7):1756-65.

2. Parry DJ, Raskin RE, Poynter JA, Ribero IB, Bajona P, Rakowski H, et al. Short and medium term outcomes of surgery for patients with hypertrophic obstructive cardiomyopathy. Ann Thorac Surg. 2015;99(4):1213-9.

3. Efthimiadis GK, Pitsis A, Pagourelias ED, Kamperidis V, Kelpis T, Meditskou S, et al. Surgical septal myectomy for hypertrophic cardiomyopathy in Greece: a single-center initial experience. Hellenic J Cardiol. 2014;55(2):132-8.

4. Rigopoulos AG, Seggewiss H. A decade of percutaneous septal ablation in hypertrophic cardiomyopathy. Circ J. 2011;75(1):28-37.

5. Butz T, Horstkotte D, Koerfer J, Langer C, Seggewiss H, Faber L. Assessment of myocardial scarring by contrast enhanced magnetic resonance imaging in a patient 11 years after percutaneous transluminal septal myocardial ablation in hypertrophic obstructive cardiomyopathy. Int J Cardiol. 2010;145(1):e3-5.

6. Jensen MK, Havndrup O, Hassager C, Helqvist S, Kelbaek H, Jorgensen E, et al. Survival and sudden cardiac death after septal ablation for hypertrophic obstructive cardiomyopathy. Scand Cardiovasc J. 2011;45(3):153-60.

7. Jensen MK, Almaas VM, Jacobsson L, Hansen PR, Havndrup O, Aakhus S, et al. Long-term outcome of percutaneous transluminal septal myocardial ablation in hypertrophic obstructive cardiomyopathy: a Scandinavian multicenter study. Circ Cardiovasc Interv. 2011;4(3):256-65.

8. Chikkabasavaiah NA, Puttegowda B, Panneerselvam A, Ananthakrishna R, Kapanigowda AP, Basavappa R. Remote infarction following percutaneous transluminal septal myocardial ablation: a report of two cases. Cardiovasc Interv Ther. 2011;26(2):142-6. experienced centers, according to the analysis result for the learning curve.

\section{Author contributions}

Conception and design of the research, Statistical analysis, Obtaining financing, Writing of the manuscript and Critical revision of the manuscript for intellectual content: Cheng-Yang L; Acquisition of data and Analysis and interpretation of the data: Cheng-Yang $L$ and Yun-Qi S.

\section{Potential Conflict of Interest}

No potential conflict of interest relevant to this article was reported.

\section{Sources of Funding}

There were no external funding sources for this study.

\section{Study Association}

This study is not associated with any thesis or dissertation work.

\section{Ethics approval and consent to participate}

This study was approved by the Ethics Committee of the Hospital da Província de Liaoning. All the procedures in this study were in accordance with the 1975 Helsinki Declaration, updated in 2013. Informed consent was obtained from all participants included in the study.
9. Nemes A, Balázs E, Soliman OI, Sepp R, Csanády M, Forster T. Longterm prognostic value of coronary flow velocity reserve in patients with hypertrophic cardiomyopathy: 9-year follow-up results from SZEGED study. Heart Vessels. 2009;24(5):352-6.

10. Gersh BJ, Maron BJ, Bonow RO, Dearani JA, Fifer MA, Link MS, et al. 2011 ACCF/AHA Guideline for the Diagnosis and Treatment of Hypertrophic Cardiomyopathy: a report of the American College of Cardiology Foundation/American Heart Association Task Force on Practice Guidelines. Developed in collaboration with the American Association for Thoracic Surgery, American Society of Echocardiography, American Society of Nuclear Cardiology, Heart Failure Society of America, Heart Rhythm Society, Society for Cardiovascular Angiography and Interventions, and Society of Thoracic Surgeons. J Am Coll Cardiol. 2011;58(25):e212-60.

11. Caselli S, Maron MS, Urbano-Moral JA, Pandian NG, Maron BJ, Pelliccia A. Differentiating left ventricular hypertrophy in athletes from that in patients with hypertrophic cardiomyopathy. Am J Cardiol. 2014;114(9):1383-9.

12. Rosella LC, Lebenbaum M, Fitzpatrick T, Zuk A, Booth GL. Prevalence of Prediabetes and Undiagnosed Diabetes in Canada (2007-2011) According to Fasting Plasma Glucose and HbA1c Screening Criteria. Diabetes Care. 2015;38(7):1299-305

13. Moon J, Cho IJ, Shim CY, Ha JW, Jang Y, Chung N, et al. Abnormal myocardial capillary density in apical hypertrophic cardiomyopathy can be assessed by myocardial contrast echocardiography. Circ J. 2010;74(10):2166-72.

14. Madsen LH, Lund T, Grieg Z, Nygaard S, Holmvang L, Jurlander B, et al. Cardiac troponin I degradation in serum of patients with hypertrophic obstructive cardiomyopathy undergoing percutaneous septal ablation. Cardiology. 2009;114(3):167-73. 
15. Gomes OM, Coelho AA, Osterne EC, Abrantes RD. Coronary morphology and conduction system disturbance induced by therapeutic embolization of the coronary septal artery. Heart Surg Forum. 2010;13(1):E45-8.

16. Faber L, Welge D, Fassbender D, Schmidt HK, Horstkotte D, Seggewiss H. Percutaneous septal ablation for symptomatic hypertrophic obstructive cardiomyopathy: managing the risk of procedure-related AV conduction disturbances. Int J Cardiol. 2007;119(2):163-7.

17. El-Jack SS, Nasif M, Blake JW, Dixon SR, Grines CL, O'Neill WW. Predictors of complete heart block after alcohol septal ablation for hypertrophic cardiomyopathy and the timing of pacemaker implantation. J Interv Cardiol. 2007;20(1):73-6.

18. van der Lee C, Scholzel B, ten Berg JM, Geleijnse ML, Idzerda $\mathrm{HH}$, van Domburg RT, et al. Usefulness of clinical, echocardiographic, and procedural characteristics to predict outcome after percutaneous transluminal septal myocardial ablation. Am J Cardiol. 2008;101(9):1315-20.

19. Veselka J, Zemánek D, Tomasov P, Duchonová R, Linhartová K. Alcohol septal ablation for obstructive hypertrophic cardiomyopathy: ultra-low dose of alcohol (1 ml) is still effective. Heart Vessels. 2009;24(1):27-31.

20. Gersh BJ, Maron BJ, Bonow RO, Dearani JA, Fifer MA, Link MS, et al. 2011 $\mathrm{ACCF} / \mathrm{AHA}$ guideline for the diagnosis and treatment of hypertrophic cardiomyopathy: executive summary: a report of the American College of Cardiology Foundation/American Heart Association Task Force on Practice Guidelines. Circulation. 2011;124(24): 2761-96.

21. Qiao SB, Yuan JS. How to improve the safety of percutaneous transluminal septal myocardial ablation. Zhonghua Xin Xue Guan Bing Za Zhi. 2011;39(3):193-5. 